\title{
Call for vigilance - red flags in systemic lupus erythematous
}

Badar Hasan, $\mathrm{MD}^{1}$, Talal Asif, $\mathrm{MD}^{1}$, Maryam Hasan, $\mathrm{MD}^{2}$, Amr Edrees, $\mathrm{MD}^{1}$

\section{Author Affiliations:}

1. University of Missouri - Kansas City, Kansas City, Missouri

2. NYU School of Medicine, Brooklyn Campus, Brooklyn, New York

The authors have no financial disclosures to declare and no conflicts of interest to report.

\section{Corresponding Author:}

Badar Hasan, MD

University of Missouri - Kansas City

Kansas City, Missouri

Email: hsn.badar@gmail.com 


\section{Abstract}

Systemic Lupus Erythematous (SLE) is a multisystem autoimmune disease. It has been identified as the underlying cause for death in an average 1,034 deaths from 2000 and 2014. Our cases highlight two rare but life-threatening complications of SLE; catastrophic antiphospholipid syndrome (CAPS) and diffuse alveolar hemorrhage (DAH) with mortality as high as 50-90\%. Both cases presented with respiratory symptoms, required meticulous monitoring in ICU and were initially treated with broad spectrum antibiotics. However, unlike pneumonia, these patients required immunosuppressive and plasmapheresis leading to clinical improvement.

\section{Keywords}

Systemic Lupus Erythematous, Catastrophic Antiphospholipid Syndrome, Diffuse Alveolar Hemorrhage

\section{Introduction}

Systemic lupus erythematous (SLE) is an autoimmune disease affecting multiple organs. The prevalence of systemic lupus erythematous is reported between 20-150 cases per 100,000. ${ }^{1}$ According to one estimate there is a prevalence of 161,000 with definite SLE and 322,000 with probable SLE in the general population. ${ }^{2}$ It was identified as the underlying cause of death for an average 1,034 deaths between 2000 and 2014. ${ }^{3}$ SLE can prove fatal due to disease activity or concurrent infections. We present two rare but life-threatening complications of SLE: catastrophic antiphospholipid syndrome (CAPS) and diffuse alveolar hemorrhage (DAH), both of which can have a mortality as high as $50-90 \%$. $^{4,5}$

\section{First Case Description}

A 39-year-old African-American female presented with soreness of bilateral lower extremities, more pronounced proximally, with difficulty in ambulation for the past four weeks. Vitals on admission were significant for low grade fever $(100.5 \mathrm{~F})$ and oxygen saturation $99 \%$ on $2 \mathrm{~L}$ oxygen. Physical exam was significant for macular rash on bilateral lower extremities and decreased breath sounds in bilateral lung bases.

Pertinent labs at the time of admission:

\begin{tabular}{|l|l|}
\hline LAB VALUES & REFERENCE RANGES \\
\hline WBC: $20.0 / \mathrm{mm} 3$ & $4.30-10.80 / \mathrm{mm} 3$ \\
\hline Hemoglobin: 9.6 (at baseline) & $12-16 \mathrm{~g} / \mathrm{dl}$ \\
\hline Platelets: 484 & $150-40010^{\wedge} 3 / \mathrm{cmm}$ \\
\hline Creatinine Kinase: 1121 & $14-54 \mathrm{U} / \mathrm{L}$ \\
\hline CRP: 23.9 & $0-1 \mathrm{mg} / \mathrm{dl}$ \\
\hline ESR: 71 & $1-20 \mathrm{~mm} / \mathrm{hr}$ \\
\hline
\end{tabular}

Chest Imaging showed left postero-basal infiltrate and developing right infiltrate (Figure 1) and pericardial effusion (Figure 2) 


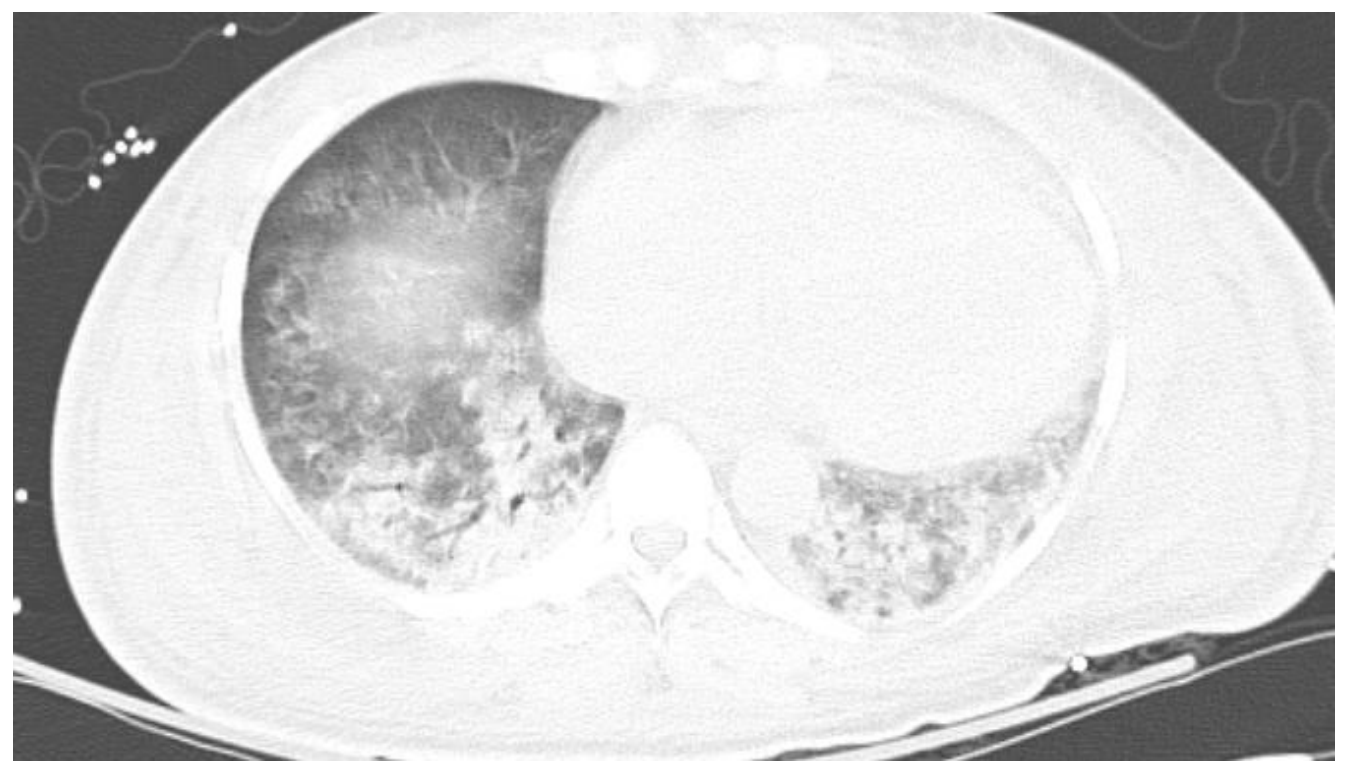

Figure 1: CT chest with and without contrast: Diffuse reticular nodular infiltrates and scattered patchy ground glass infiltrates.

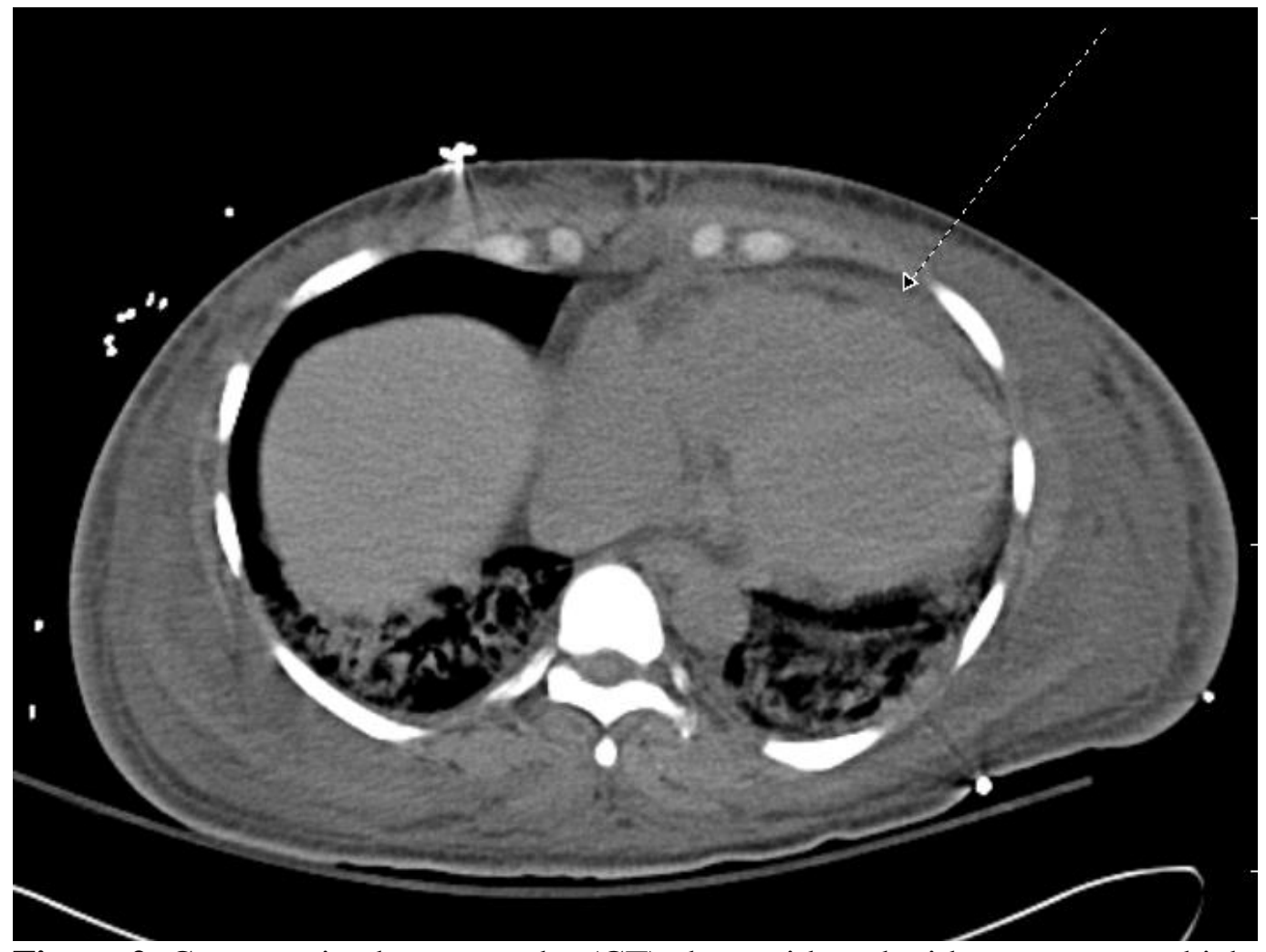

Figure 2: Computerized tomography (CT) chest with and without contrast which shows mild cardiomegaly with pericardial effusion (dotted arrow). 
On the second day of hospitalization the patient was transferred to the intensive care unit (ICU) because of increased shortness of breath with continuous fever and subsequently required intubation for hypoxia. Shortly after that she developed hypotension requiring brief pressor support. The patient started to develop discoloration of her distal digits which was initially thought to be a result of hypo perfusion during the hypotensive crisis. The working diagnosis at this point was pneumonia causing sepsis and thus antibiotic coverage was broadened. During the ICU stay, the patient also developed acute renal failure with decreased urine output requiring intermittent hemodialysis. Echo demonstrated pericardial effusion with tamponade, which was subsequently drained and $600 \mathrm{ml}$ of serous fluid was removed. Pericardial fluid was positive for antinuclear antibodies (ANA). Her blood work up also came back positive for ANA (titer levels of 1:600) with other notable findings being low complement levels and positive antiphospholipid antibody and SSA Ab.

At this point patient's infectious work up including sputum, blood culture and bronchoalveolar lavage which remained negative and patient's respiratory failure was attributed to acute lupus pneumonitis. She underwent renal biopsy which demonstrated lupus nephritis, WHO class IIB and III.

Antibiotics were discontinued and per rheumatology she was started on 1g/day of methyl prednisone, IV Cytoxan and heparin drip. After a prolonged hospital stay she was weaned off from the ventilator and was maintained on prednisone for suppression of her SLE and anticoagulation in context of digit hypo perfusion. Two months later she was readmitted and underwent right thumb, index, middle, small finger and left second and fifth toe amputation. Microscopic examination of upper extremity amputation revealed thrombi in the medium-size arteries.

In light of histological manifestation of vessel occlusion, presence of antiphospholipid antibody, and rapid progression of symptoms with multiple digit involvement led to a diagnosis of catastrophic antiphospholipid syndrome. ${ }^{4}$ The patient subsequently was maintained on lifelong anticoagulation and immunosuppression.

\section{Second Case Description}

A 26-year-old African-American female with a past medical history significant for asthma, seasonal allergies and recent diagnosis of SLE (mainly mucocutaneous and inflammatory arthritis) presented with a two-week history of progressive dyspnea, hemoptysis and nocturnal fevers.

The patient reported that initially she had dyspnea at rest with occasional blood-tinged sputum but now developed hemoptysis with accompanying fevers at night time. She denied any sick contacts but worked at a daycare center. On presentation, she was tachypneic, requiring 3 L of oxygen. She was afebrile and normotensive. Physical exam was significant for anterior cervical and submandibular lymphadenopathy. Chest auscultation was remarkable for bilateral rhonchi, left lower lobe wheezing with skin findings positive for malar and discoid rash noted on the face and ear respectively. 
Pertinent Labs at the time of admission:

\begin{tabular}{|l|l|}
\hline LAB VALUES & REFERENCE RANGES \\
\hline $\begin{array}{l}\text { Hemoglobin: } 5.4 \\
\text { (Baseline-11-12) }\end{array}$ & $12-16 \mathrm{~g} / \mathrm{dl}$ \\
\hline ANA: $1: 10,240$ & $<1: 40$ \\
\hline ESR: $>121$ & $1-20 \mathrm{~mm} / \mathrm{hr}$ \\
\hline Complement C4: $<2$ & $10-40 \mathrm{mg} / \mathrm{dl}$ \\
\hline Complement C3: 66 & $90-180 \mathrm{mg} / \mathrm{dl}$ \\
\hline
\end{tabular}

Computerized tomography angiography (CTA) of chest was significant for diffuse adenopathy and ground glass opacities as seen in Figure 3.

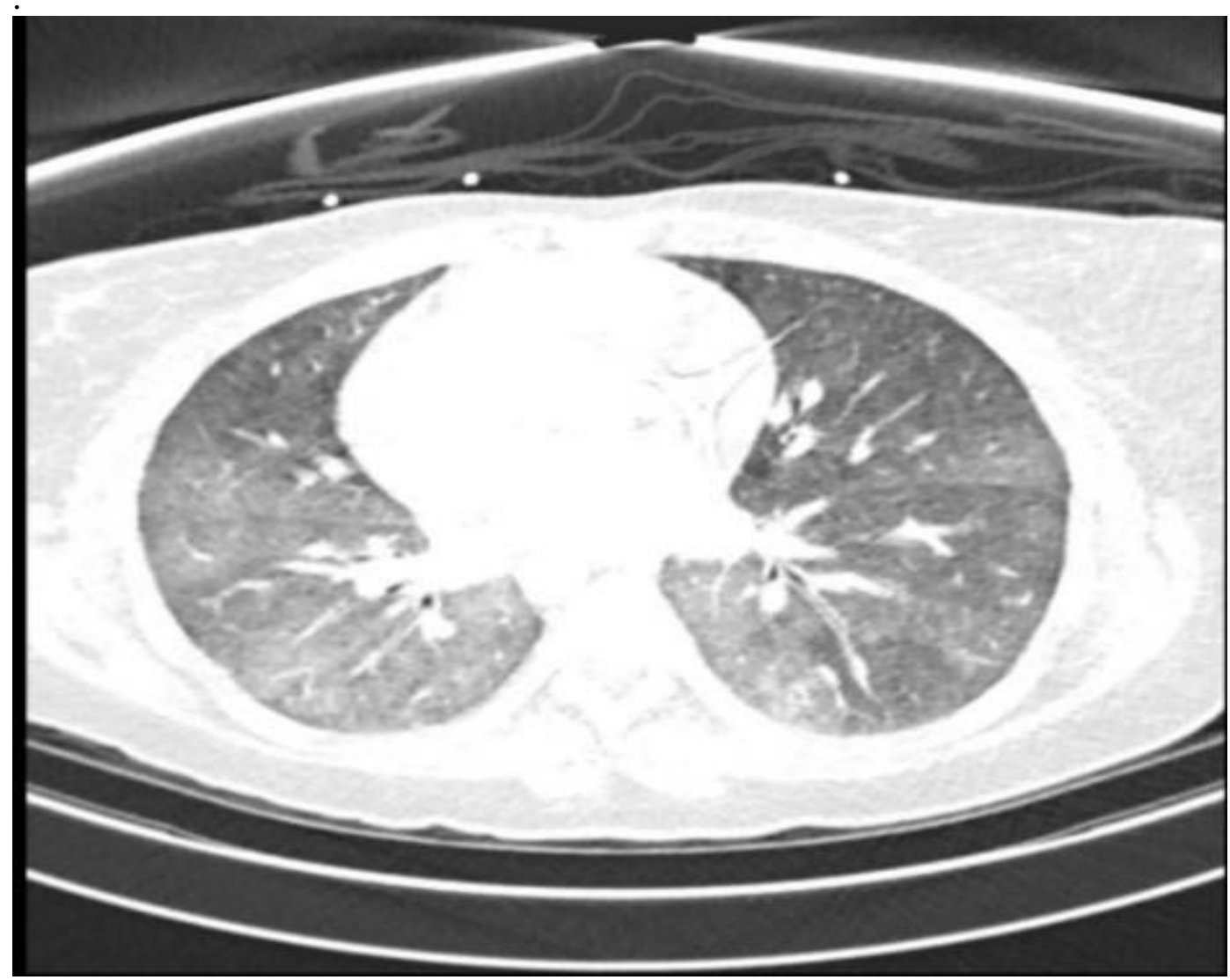

Figure 3: CT Angiography of chest: Confluent diffuse bilateral ground glass opacities/confluent airspace consolidations with a subtle dependent gradient of airspace opacity.

The patient was admitted to the ICU and started on broad-spectrum antibiotics. She underwent bronchoalveolar lavage which was consistent with diffuse alveolar hemorrhage (DAH). Rheumatology was consulted and the patient was promptly started on a five-day course of 1 $\mathrm{g} /$ day methyl prednisone (along with PCP prophylaxis and IV immunoglobulin). Four days later 
she had worsening cough and her respiratory function declined, requiring intubation. Femoral dialysis catheter was placed and plasmapheresis initiated for 5 days along with rituximab and cyclophosphamide. During her course of admission mycoplasma IgM antibody (1312 U/ml) and fungi tell came back positive. Infectious diseases was consulted and antimicrobial coverage was augmented with voriconazole. On the ninth day of admission she was extubated, antibiotics and antifungal were discontinued as infectious and blood workup (except mycoplasma) remained negative. Mycoplasma pneumonia treatment was completed with 7-day course of antibiotic coverage (which patient was on since admission). Subsequently she was transferred to the floor with continued improvement in her oxygen requirements. She was maintained on PCP prophylaxis with her prednisone taper and ultimately discharged on her home regimen of hydroxychloroquine with outpatient follow-up.

\section{Discussion}

These cases highlight that not every patient who presents with respiratory failure and lung infiltrates (as seen in figures 2 and 3) may have pneumonia requiring broad-spectrum antibiotics. Moreover, these lethal complications can be the first manifestation of SLE itself as illustrated in the case of catastrophic antiphospholipid syndrome (case one). Both cases required close monitoring in an intensive care setting and treatment with immunosuppressive agents:

corticosteroids and cyclophosphamide. Additionally, the patient in case two also required plasma exchange. ${ }^{4}$ Anticoagulants are required in CAPS whereas extracorporeal membrane oxygenation (ECMO) can be used in severe cases of DAH. ${ }^{6}$ A high index of suspicion, early recognition of symptoms and treatment with immunosuppressive agents remain the keys to improve quality of care, prevent progression of organ failure and hence prevent mortality in CAPS and DAH. 


\section{References}

1. Lawrence RC, Helmick CG, Arnett FC, Deyo RA, Felson DT, Giannini EH, Heyse SP, Hirsch R, Hochberg MC, Hunder GG, Liang MH, Pillemer SR, Steen VD, Wolfe F. Estimates of the prevalence of arthritis and selected musculoskeletal disorders in the United States. Arthritis \& Rheumatism. 1998;41(5):778-99.

2. Helmick CG, Felson DT, Lawrence RC, Gabriel S, Hirsch R, Kwoh CK, Liang MH, Kremers HM, Mayes MD, Merkel PA, Pillemer SR, Reveille JD, Stone JH. Estimates of the prevalence of arthritis and other rheumatic conditions in the United States: Part I. Arthritis \& Rheumatism. 2007;58(1):15-25.

3. CDC WONDER FAQ Help Contact Us WONDER Search [Internet]. Centers for Disease Control and Prevention. Centers for Disease Control and Prevention; [cited 2017Apr9]. Available from: https://wonder.cdc.gov/cmf-icd10.html

4. Kazzaz NM, Mccune WJ, Knight JS. Treatment of catastrophic antiphospholipid syndrome. Current Opinion in Rheumatology. 2016;28(3):218-27.

5. Ednalino C, Yip J, Carsons SE. Systematic Review of Diffuse Alveolar Hemorrhage in Systemic Lupus Erythematosus. JCR: Journal of Clinical Rheumatology. 2015;21(6):305-10.

6. Patel JJ, Lipchik RJ. Systemic Lupus-Induced Diffuse Alveolar Hemorrhage Treated With Extracorporeal Membrane Oxygenation. Journal of Intensive Care Medicine. 2014;29(2):104-9. 